\title{
On Contractions of Three-Dimensional Complex Associative Algebras
}

\author{
Mohammed NF ${ }^{1,3}$, Rakhimov IS $^{1,2 *}$ and Sh Said Husain $\mathrm{K}^{1,2}$ \\ ${ }^{1}$ Department of Mathematics, Faculty of Science, Institute for Mathematical Research, UPM, Malaysia \\ ${ }^{2}$ Laboratory of Cryptography, Analysis and Structure, Institute for Mathematical Research (INSPEM), UPM, Malaysia \\ ${ }^{3}$ Department of Mathematics, College of Education for Pure Sciences/Ibn-AL-Haithem, University of Baghdad, Iraq
}

\begin{abstract}
Contraction is one of the most important concepts that motivated by numerous applications in different fields of physics and mathematics. In this work, the contractions of complex associative algebras are considered. We focus on the variety $A_{3}(\mathbb{C})$ of all complex associative algebras of dimension three (including nonunital). Various contractions criteria are collected and new criteria are proposed to test the possible existence of contraction for each pair of associative algebras. One of the main tools is the use of the low-dimensional cohomology groups of these algebras. As a result, we prove that the variety $A_{3}(\mathbb{C})$ has seven irreducible components, two of dimension 5 , four of dimension 7 and one of dimension 9.
\end{abstract}

\section{Introduction}

The notion of contractions was first introduced by Segal [1] and Inonu [2] for Lie algebras. According to references, the contractions can be divided into two major categories. The first one is more physical that deals with the applications of contractions. Another one is pure algebraic that is mainly oriented to the abstract algebraic structure and mathematical background. For associative algebras, Gabriel [3] studied the irreducible components of the algebraic variety of 4-dimensional unital associative algebras. Mazzola's paper [4] concerns unital associative algebras of dimension five. Classification of lowdimensional nilpotent rigid associative algebras and the description of the irreducible components have been treated by Makhlouf $[5,6]$.

The main purpose of this work is to study the variety of all 3-dimensional complex associative algebras. In the paper, we deal with the algebraic point of view of the contractions. We study orbit closures of the variety of complex associative algebras of dimension three. The paper is organized as follows. Some notations on associative algebras, degeneration, rigidity and contractions of associative algebras are given in Section 2. In Section 3, we list some important invariance arguments for contractions. Calculation and collection of invariance arguments are adduced to conclude the possible existence of contractions for an arbitrary pair of associative algebras in Section 4.

\section{Preliminaries}

In this section, we recall some terminology that are used in the paper. Let $A=(V, \lambda)$ be an algebra of dimension $n$ with an underlying vector space $V$ over a field $\mathbb{K}$ and product $\lambda: V \times V \rightarrow V$. Let $g:(0,1] \rightarrow G L(V)$ be a continuous function. More precisely for any $t \in(0,1]$, a nonsingular linear operator $g_{t}$ on $V$ is assigned. A parameterized family of new isomorphic to $A=(V, \lambda)$ algebra structures on $V$ is determined as follows:

$$
\lambda_{t}(x, y)=g_{t}^{-1} \lambda\left(g_{t}(x), g_{t}(y)\right), \quad x, y \in V
$$

\section{Definition 2.1}

If for any $x, y \in V$, the limit $\lim _{t \rightarrow+0} \lambda_{t}(x, y)=\lambda_{0}(x, y)$ exists then algebraic structure $\lambda_{0}$ is called a contraction of $\lambda$.

A contraction $B$ of $A$ to algebra $B$ is called trivial if $B$ is abelian and improper if $B$ is isomorphic to $A$. Consider an $n^{3}$-dimensional vector space $\operatorname{Hom}(V \otimes V, V)$ formed by bilinear maps $V \times V \rightarrow V$, where $V$ is an $n$-dimensional vector space over an algebraically closed field $\mathbb{K}(\operatorname{char} \mathbb{K}=0)$ denoted by $A \lg _{n}(\mathbb{K})$. An algebra $A=(V, \lambda)$ is given as an element $\lambda(A)$ of $A \lg _{n}(\mathbb{K})$ through the linear mapping $\lambda: V \otimes V \rightarrow V$. The linear reductive group $G L_{n}(\mathbb{K})$ acts on $A g_{n}(\mathbb{K})$ by

$$
(g * \lambda)(x, y)=g\left(\lambda\left(g^{-1}(x), g^{-1}(y)\right)\right) .
$$

Under this action, two algebras $A$ and $B$ belong to the same orbit if and only if they are isomorphic. Moreover, we say that algebra $A$ degenerates to algebra $B$, if $B$ lies in Zariski closure of the orbit of $A$. This is denoted by $A \underset{\operatorname{deg}}{\longrightarrow} B$.

\section{Definition 2.2}

Let $A$ be an algebra over a field $\mathbb{K}$. We call $A$ an associative algebra if its bilinear mapping $\lambda$ satisfies the following condition

$$
\lambda(\lambda(x, y), z)=\lambda(x, \lambda(y, z)), \quad x, y, z \in V .
$$

Let $A_{n}(\mathbb{K})$ be the set of all associative algebra structures on $n$-dimensional space over a field $\mathbb{K}$. The set $A_{n}(\mathbb{K})$ is an algebraic subset of the affine variety $\operatorname{Alg}_{n}(\mathbb{K})$. For a fixed basis $\left\{e_{1}, e_{2}, \ldots, e_{n}\right\}$ of the vector space $V$, the multiplication table of $A$ on this basis is given as a point $\left(\gamma_{i j}^{k}\right) \in \mathbb{K}^{n^{3}}$ as follows

$$
\lambda\left(e_{i}, e_{j}\right)=\sum_{k=1}^{n} \gamma_{i j}^{k} e_{k}, \quad i, j=1, \cdots, n .
$$

Let $A$ be an associative $\mathbb{K}$-algebra, $D$ be an $A$-bimodule and $\Phi: A^{P} \rightarrow D$ be a multilinear mapping. The set of all multilinear mappings from $A^{P}$ to $D$ is called $p$-dimensional cochain of $A$ and denoted by $C^{p}(A, D)$. The coboundary homomorphism is a mapping $\delta^{(p)}$ from $C^{p}(A, D)$ to $C^{p+1}(A, D)$ given by

*Corresponding author: Rakhimov IS, Laboratory of Cryptography, Analysis and Structure, Institute for Mathematical Research (INSPEM), UPM, Malaysia, Tel: 038946 6831; E-mail: risamiddin@gmail.com

Received July 06, 2017; Accepted September 29, 2017; Published October 10 2017

Citation: Mohammed NF, Rakhimov IS, Said Husain SK (2017) On Contractions of Three-Dimensional Complex Associative Algebras. J Generalized Lie Theory Appl 11: 282. doi: 10.4172/1736-4337.1000282

Copyright: ( 2017 Mohammed NF, et al. This is an open-access article distributed under the terms of the Creative Commons Attribution License, which permits unrestricted use, distribution, and reproduction in any medium, provided the original author and source are credited. 


$$
\begin{aligned}
& \left(\delta^{(p)} \Phi\right)\left(x_{1}, x_{2}, \cdots, x_{p+1}\right)=x_{1} \Phi\left(x_{2}, \cdots, x_{p+1}\right)+\sum_{i=1}^{p}(-1)^{i} \\
& \Phi\left(x_{1}, \cdots, x_{i} x_{i+1}, \cdots, x_{p+1}\right)+(-1)^{p+1} \Phi\left(x_{1}, \cdots, x_{p}\right) x_{p+1} .
\end{aligned}
$$

The kernel of the coboundary operator is denoted by $Z{ }^{p}(A, D)$ whose elements are called $p$-cocycles with values in $D$. The elements of the image of $\delta^{(p-1)}$ denoted by $B^{p}(A, D)$ are called $p$-coboundaries with values in $D$. The quotient space:

$$
H^{p}(A, D)=Z^{p}(A, D) / B^{p}(A, D)
$$

is called the cohomology space (group) of $A$ of degree $p$. In this paper, we will consider a particular case that $D=A$ as $A$-bimodule.

\section{Definition 2.3}

An associative algebra $A$ is called geometrically rigid whenever its orbit is Zariski open in $A_{n}(\mathbb{K})$ and called algebraically rigid if the second cohomology group $H^{2}(A, A)$ is trivial [7].

\section{Invariance Arguments}

In this section, we list some invariance arguments which are helpful for studying the variety of a given class of algebras. Let $A$ be an associative algebras over a field $\mathbb{K}$. We define:

- $A^{k}=\lambda\left(A^{k-1}, A\right)$ - the $k$-th degree of $A$, where $k \in \mathbb{N}$;

- $R(A)=\{x \in A \mid \lambda(A, x)=0\}$ - the right annihilator of $A$;

- $L(A)=\{x \in A \mid \lambda(x, A)=0\}$ - the left annihilator of $A$;

- $Z(A)=\{x \in A \mid \lambda(x, y)=\lambda(y, x), \forall y \in A\}$ - the center of $A$;

- $\operatorname{Aut}(A)=\{d: A \rightarrow A \mid d(\lambda(x, y))=\lambda(d(x), d(y)), \forall x, y \in A\} \quad$ - the group of automorphisms of $A$;

- $S A(A)$ - the maximal abelian subalgebra of $A$;

- $\operatorname{Com}(A)$ - the maximal commutative subalgebra of $A$;

- $O(A)$ - the orbit of $A$;

- $\operatorname{Der}(A)=\{d \in \operatorname{End}(A) \mid d(\lambda(x, y))=\lambda(d(x), y)+\lambda(x, d(y)), \forall x, y \in A\}$ - the algebra of derivations of $A$;

- $r_{n}(A)$-the nilpotency rank of an associative algebra $A$;

- $H^{i}(A, A)$ - the $i^{\text {th }}$ cohomology group of $A$;

- $Z^{2}(A, A)$ - the 2-cocycles of an associative algebra $A$;

- $\operatorname{Der}_{(\alpha, \beta, \gamma)}(A)=\{d \in \operatorname{End}(A) \mid \alpha d(\lambda(x, y))=\beta \lambda(d(x), y)+\gamma \lambda(x, d(y), \forall x, y \in A\}$ is the space of $(\alpha, \beta, \gamma)$-derivations of $A$, for fixed $\alpha, \beta, \gamma \in \mathbb{K}$.

The following theorem is very useful to study the irreducible components of the subvariety $A_{n}(\mathbb{K})$ of $A \lg _{n}(\mathbb{K})[8,9]$.

\section{Theorem 3.1}

The the following subsets of $A_{n}(\mathbb{K})$ are closed relative to the Zariski topology for any $r, s \in \mathbb{N}: 0.60 t w 0.60 t w$

- (1) $\left\{A \in A_{n}(\mathbb{K}) \mid \operatorname{dim}_{\mathbb{K}} A^{r} \leq s\right\}$;

- (3) $\left\{A \in A_{n}(\mathbb{K}) \mid \operatorname{dim}_{\mathbb{K}} L(A) \geq r\right\}$;

- (5) $\left\{A \in A_{n}(\mathbb{K}) \mid \operatorname{dim}_{\mathbb{K}} \operatorname{Com}(A) \geq r\right\}$;

- (7) $\left\{A \in A_{n}(\mathbb{K}) \mid \operatorname{dim}_{\mathbb{K}} \operatorname{Aut}(A)>r\right\}$;

- (9) $\left\{A \in A_{n}(\mathbb{K}) \mid \operatorname{dim}_{\mathbb{K}} \operatorname{Der}(A)>r\right\}$;

- (11) $\left\{A \in A_{n}(\mathbb{K}) \mid \operatorname{dim}_{\mathbb{K}} H(A, A) \geq r\right\}$;
- (13) $\left\{A \in A_{n}(\mathbb{K}) \mid \operatorname{dim}_{\mathbb{K}} \operatorname{Der}_{(\alpha, \beta, \gamma)}(A) \geq r\right\}$.

- (2) $\left\{A \in A_{n}(\mathbb{K}) \mid \operatorname{dim}_{\mathbb{K}} R(A) \geq r\right\}$;

- (4) $\left\{A \in A_{n}(\mathbb{K}) \mid \operatorname{dim}_{\mathbb{K}} Z(A) \geq r\right\}$;

-(6) $\left\{A \in A_{n}(\mathbb{K}) \mid \operatorname{dim}_{\mathbb{K}} \mathrm{SA}(A) \geq r\right\}$;

- (8) $\left\{A \in A_{n}(\mathbb{K}) \mid \operatorname{dim}_{\mathbb{K}} O(A)<r\right\}$;

-(10) $\left\{A \in A_{n}(\mathbb{K}) \mid r_{n}(A) \leq r\right\}$;

- (12) $\left\{A \in A_{n}(\mathbb{K}) \mid \operatorname{dim}_{\mathbb{K}} Z^{2}(A, A) \geq r\right\}$;

The proof of $1-4$ is the same to that of Lie algebras $[10,11]$. For the parts 5 and 6 , the proof is obtained by the following significant fact: let $N$ be a Zariski closed subset of $A_{n}(\mathbb{K})$ and $A_{1}, A_{2}$ in $A_{n}(\mathbb{K})$. If $A_{1}$ lies in $N$ and $A_{1} \rightarrow A_{2}$ then $A_{2}$ also lies in $A$. More precisely, the subset $N$ is not $G L_{n}(\mathbb{K})$-setwise stabilizer. However, it is $B$-setwise stabilizer, where $B$ is the Borel subgroup $G L_{n}(\mathbb{K})$ - made up of upper triangular matrices. The statements 7,8 and 9 are equivalent because of the following relation between the dimensions of $G L_{n}(\mathbb{K})$-- orbits, automorphism's groups and derivation algebras.

$$
\operatorname{dim} O(A)=n^{2}-\operatorname{dim} \operatorname{Aut}(A)=n^{2}-\operatorname{dim} \operatorname{Der}(A)
$$

The proof of 10 is directly coming behind the following fact: let $A$ be an associative algebra. We define the lower central series:

$A^{1} \supset A^{2} \supset \cdots \supset A^{k+1} \supset \cdots$

If $A$ is a nilpotent associative algebra then it has nilpotency rank denoted by $r_{n}(A)$, i.e., it is a minimal positive integer $l$ such that $A^{l}=0$. It is not hard to see that if $A_{1}$ degenerates to $A_{2}$ then $\operatorname{dim} A_{1}^{k+1} \geq \operatorname{dim} A_{2}^{k+1}$. The proof of 11 and 12 are the same of Lie algebras [12].

The next corollary is used to reject existence of degenerations for each pair of associative algebras $A$ and $B$.

\section{Corollary 3.1}

If an algebra $A$ degenerates to an algebra $B$. Then the following conditions are valid:

- (1) $\operatorname{dim}_{\mathbb{K}} A^{r} \geq \operatorname{dim}_{\mathbb{K}} B^{r}$ for some $r$,

- (3) $\operatorname{dim}_{\mathbb{K}} L(A) \leq \operatorname{dim}_{\mathbb{K}} L(B)$,

- (5) $\operatorname{dim}_{\mathbb{K}} \operatorname{Com}(A) \leq \operatorname{dim}_{\mathbb{K}} \operatorname{Com}(B)$,

- (7) $\operatorname{dim}_{\mathbb{K}} \operatorname{Aut}(A)<\operatorname{dim}_{\mathbb{K}} \operatorname{Aut}(B)$,

- (11) $\operatorname{dim}_{\mathbb{K}} \mathrm{H}^{i}(A, A) \leq \operatorname{dim}_{\mathbb{K}} \mathrm{H}^{i}(B, B)$,

- (11) $\operatorname{dim}_{\mathbb{K}} \mathrm{H}^{i}(A, A) \leq \operatorname{dim}_{\mathbb{K}} \mathrm{H}^{i}(B, B)$,

- (13) $\operatorname{dim}_{\mathbb{K}} \operatorname{Der}_{(\alpha, \beta, \gamma)}(A) \leq \operatorname{dim}_{\mathbb{K}} \operatorname{Der}_{(\alpha, \beta, \gamma)}(B)$.

- (2) $\operatorname{dim}_{\mathbb{K}} R(A) \leq \operatorname{dim}_{\mathbb{K}} R(B)$,

- (4) $\operatorname{dim}_{\mathbb{K}} Z(A) \leq \operatorname{dim}_{\mathbb{K}} Z(B)$,

- (6) $\operatorname{dim}_{\mathbb{K}} \mathrm{SA}(A) \leq \operatorname{dim}_{\mathbb{K}} \mathrm{SA}(B)$,

- (8) $\operatorname{dim}_{\mathbb{K}} O(A)>\operatorname{dim}_{\mathbb{K}} O(B)$,

- (10) $r_{n}(A) \geq r_{n}(B)$

- (12) $\operatorname{dim}_{\mathbb{K}} Z^{2}(A, A) \leq \operatorname{dim}_{\mathbb{K}} Z^{2}(B, B)$.

In the sequel, all algebras suppose to be over the field of complex numbers $\mathbb{C}$. 


\section{The Variety of Complex Associative Algebras of Dimension Three}

In this section, we recall the complete list of non-isomorphic classes of three-dimensional complex associative algebras, which was obtained in Rikhsiboev et al. [13] to study the subvariety $A_{3}(\mathbb{C})$ of $A \lg _{3}(\mathbb{C})$.

\section{Theorem 4.1}

Any 3-dimensional complex associative algebra $A$ is isomorphic to one of the following pairwise non-isomorphic algebras.

$$
\begin{array}{lll}
A s_{3}^{1}: & e_{1} e_{3}=e_{2}, \quad e_{3} e_{1}=e_{2} ; \\
A s_{3}^{2}(\alpha): & e_{1} e_{3}=e_{2}, \quad e_{3} e_{1}=\alpha e_{2}, \quad \alpha \in \mathbb{C} \backslash\{1\} ; \\
A s_{3}^{3}: \quad e_{1} e_{1}=e_{2}, \quad e_{1} e_{2}=e_{3}, \quad e_{2} e_{1}=e_{3} ; \\
A s_{3}^{4}: \quad e_{1} e_{3}=e_{2}, \quad e_{2} e_{3}=e_{2}, \quad e_{3} e_{3}=e_{3} ; \\
A s_{3}^{5}: \quad e_{2} e_{3}=e_{2}, \quad e_{3} e_{1}=e_{1}, \quad e_{3} e_{3}=e_{3} ; \\
A s_{3}^{6}: \quad e_{3} e_{1}=e_{2}, \quad e_{3} e_{2}=e_{2}, \quad e_{3} e_{3}=e_{3} ; \\
A s_{3}^{7}: \quad e_{1} e_{2}=e_{1}, \quad e_{2} e_{2}=e_{2}, \quad e_{3} e_{1}=e_{1}, \quad e_{3} e_{3}=e_{3} ; \\
A s_{3}^{8}: \quad e_{1} e_{3}=e_{1}, \quad e_{2} e_{3}=e_{2}, \quad e_{3} e_{1}=e_{1}, \quad e_{3} e_{3}=e_{3} ; \\
A s_{3}^{9}: \quad e_{2} e_{3}=e_{2}, \quad e_{3} e_{1}=e_{1}, \quad e_{3} e_{2}=e_{2}, \quad e_{3} e_{3}=e_{3} ; \\
A s_{3}^{10}: \quad e_{1} e_{3}=e_{1}, \quad e_{2} e_{3}=e_{2}, \quad e_{3} e_{1}=e_{1}, \quad e_{3} e_{2}=e_{2}, \quad e_{3} e_{3}=e_{3} ; \\
A s_{3}^{11}: \quad e_{1} e_{3}=e_{2}, \quad e_{2} e_{3}=e_{2}, \quad e_{3} e_{1}=e_{2}, \quad e_{3} e_{2}=e_{2}, \quad e_{3} e_{3}=e_{3} ; \\
A s_{3}^{12}: \quad e_{1} e_{1}=e_{2}, \quad e_{1} e_{3}=e_{1}, \quad e_{2} e_{3}=e_{2}, \quad e_{3} e_{1}=e_{1}, \quad e_{3} e_{2}=e_{2}, \quad e_{3} e_{3}=e_{3} ; \\
A s_{3}^{13}: \quad e_{1} e_{1}=e_{1}, \quad e_{2} e_{2}=e_{2}, \quad e_{3} e_{3}=e_{3} ; \\
A s_{3}^{14}: \quad e_{1} e_{2}=e_{1}, \quad e_{2} e_{1}=e_{1}, \quad e_{2} e_{2}=e_{2}, \quad e_{3} e_{3}=e_{3} ; \\
A s_{3}^{15}: \quad e_{1} e_{2}=e_{1}, \quad e_{2} e_{2}=e_{2}, \quad e_{3} e_{3}=e_{3} ; \\
A s_{3}^{16}: \quad e_{2} e_{1}=e_{1}, \quad e_{2} e_{2}=e_{2}, \quad e_{3} e_{3}=e_{3} ; \\
A s_{3}^{17}: \quad e_{1} e_{1}=e_{2}, \quad e_{3} e_{3}=e_{3} ; \\
A s_{3}^{18}: & A b e l i a n .
\end{array}
$$

\begin{tabular}{|c|c|c|c|c|c|}
\hline IC & $\operatorname{dim}_{\mathbb{K}}(R(A))$ & $\operatorname{dim}_{\mathbb{K}}(L(A))$ & $\operatorname{dim}_{\mathbb{K}}(\operatorname{Aut}(A))$ & $\operatorname{dim}_{\mathbb{K}}(\operatorname{Com}(A))$ & $\operatorname{dim}_{\mathbb{K}}(\operatorname{Der}(A))$ \\
\hline$A s_{3}^{1}$ & 1 & 1 & 4 & 3 & 4 \\
\hline$A s_{3}^{2}$ & 1 & 1 & 4 & 2 & 4 \\
\hline$A s_{3}^{3}$ & 1 & 1 & 3 & 3 & 3 \\
\hline$A s_{3}^{4}$ & 2 & 2 & 3 & 2 & 3 \\
\hline$A s_{3}^{5}$ & 1 & 1 & 4 & 2 & 4 \\
\hline$A s_{3}^{6}$ & 2 & 2 & 3 & 2 & 3 \\
\hline$A s_{3}^{7}$ & 0 & 0 & 2 & 2 & 2 \\
\hline$A s_{3}^{8}$ & 1 & 0 & 3 & 2 & 3 \\
\hline$A s_{3}^{9}$ & 0 & 1 & 3 & 2 & 3 \\
\hline$A s_{3}^{10}$ & 0 & 0 & 4 & 3 & 4 \\
\hline$A s_{3}^{11}$ & 2 & 2 & 2 & 3 & 2 \\
\hline$A s_{3}^{12}$ & 0 & 0 & 2 & 3 & 2 \\
\hline$A s_{3}^{13}$ & 0 & 0 & 0 & 3 & 0 \\
\hline$A s_{3}^{14}$ & 0 & 0 & 1 & 3 & 1 \\
\hline$A s_{3}^{15}$ & 1 & 0 & 2 & 2 & 2 \\
\hline$A s_{3}^{16}$ & 0 & 1 & 2 & 2 & 2 \\
\hline$A s_{3}^{17}$ & 1 & 1 & 2 & 3 & 2 \\
\hline$A s_{3}^{18}$ & 3 & 3 & 9 & 3 & 9 \\
\hline
\end{tabular}

Table 1: The collected contraction invariants of three-dimensional associative algebras.
In Table 1, we assemble some contraction invariants of three-

In Table 2 below we present the values of more contraction invariants.

Table 3 contains the values of contraction invariants applying the algorithm, which was stated in ref. [16] to describe the $(\alpha, \beta, \gamma)$ derivations of three- dimensional complex associative algebras.

By using all the criteria presented above, we give in the following table all possibilities of degenerations for 3-dimensional associative algebras. The checkmark denotes that there is a degeneration $A \longrightarrow \underset{d e g}{\longrightarrow} B$. The other symbols stand for the reason why such a degeneration is impossible. Indeed, there is more than just one reason for a non-degeneration. However, we have written down only one in the table (Table 4).

According to Table 4, the algebras $A s_{3}^{2}, A s_{3}^{5}, A s_{3}^{7}, A s_{3}^{12}, A s_{3}^{13}, A s_{3}^{15}$ and , $A s_{3}^{16}$ and are geometrically rigid. More precisely, they are not degeneration of other 3-dimensional associative algebras structures. dimensional associative algebras that were obtained in refs. [14,15]. 
Citation: Mohammed NF, Rakhimov IS, Said Husain SK (2017) On Contractions of Three-Dimensional Complex Associative Algebras. J Generalized Lie Theory Appl 11: 282. doi: 10.4172/1736-4337.1000282

Page 4 of 6

\begin{tabular}{|c|c|c|c|c|c|c|c|c|}
\hline IC & $\operatorname{dim}_{\mathbb{K}}\left(\left(A^{2}\right)\right)$ & $\operatorname{dim}_{\mathbb{K}}(Z(A))$ & $\operatorname{dim}_{\mathbb{K}}(\mathrm{SA}(\mathrm{A}))$ & $\operatorname{dim}_{\mathbb{K}}(\mathrm{O}(\mathrm{A}))$ & $r_{3}(A)$ & $\operatorname{dim}_{\mathbb{K}}\left(\mathrm{H}^{1}(A, A)\right)$ & $\operatorname{dim}_{\mathbb{K}}\left(\mathrm{H}^{2}(A, A)\right)$ & $\operatorname{dim}_{\mathbb{K}}\left(Z^{2}(A, A)\right)$ \\
\hline$A s_{3}^{1}$ & 1 & 3 & 2 & 5 & 3 & 4 & 4 & 9 \\
\hline$A s_{3}^{2}$ & 1 & 1 & 2 & 5 & 3 & 2 & 3 & 8 \\
\hline$A s_{3}^{3}$ & 2 & 3 & 2 & 6 & 4 & 3 & 3 & 9 \\
\hline$A s_{3}^{4}$ & 2 & 2 & 2 & 6 & - & 1 & 2 & 8 \\
\hline$A s_{3}^{5}$ & 3 & 0 & 2 & 5 & - & 1 & 0 & 5 \\
\hline$A s_{3}^{6}$ & 2 & 2 & 2 & 6 & - & 1 & 2 & 8 \\
\hline$A s_{3}^{7}$ & 3 & 2 & 1 & 7 & - & 0 & 0 & 7 \\
\hline$A s_{3}^{8}$ & 3 & 1 & 2 & 6 & - & 1 & 1 & 7 \\
\hline$A s_{3}^{9}$ & 3 & 1 & 2 & 6 & - & 1 & 1 & 7 \\
\hline$A s_{3}^{10}$ & 3 & 3 & 2 & 5 & - & 4 & 6 & 11 \\
\hline$A s_{3}^{11}$ & 2 & 3 & 2 & 7 & - & 2 & 2 & 9 \\
\hline$A s_{3}^{12}$ & 3 & 3 & 1 & 7 & - & 2 & 0 & 7 \\
\hline$A s_{3}^{13}$ & 3 & 3 & 0 & 9 & - & 0 & 0 & 9 \\
\hline$A s_{3}^{14}$ & 3 & 3 & 1 & 8 & - & 1 & 1 & 9 \\
\hline$A s_{3}^{15}$ & 3 & 1 & 1 & 7 & - & 0 & 0 & 7 \\
\hline$A s_{3}^{16}$ & 3 & 1 & 1 & 7 & - & 0 & 0 & 7 \\
\hline$A s_{3}^{17}$ & 2 & 3 & 1 & 7 & - & 2 & 2 & 9 \\
\hline$A s_{3}^{18}$ & 0 & 3 & 3 & 0 & 2 & 9 & 27 & 27 \\
\hline
\end{tabular}

Table 2: The calculated contraction invariants of three-dimensional associative algebras.

\begin{tabular}{|c|c|c|c|c|c|c|}
\hline IC & $\operatorname{dim}_{\mathbb{K}}\left(D e r_{(1,1,0)}(A)\right)$ & $\operatorname{dim}_{\mathbb{K}}\left(\operatorname{Der}_{(1,0,1)}(A)\right)$ & $\operatorname{dim}_{\mathbb{K}}\left(\operatorname{Der}_{(1,0,0)}(A)\right)$ & $\operatorname{dim}_{\mathbb{K}}\left(\operatorname{Der}_{(0,1,1)}(A)\right)$ & $\operatorname{dim}_{\mathbb{K}}\left(\operatorname{Der}_{(0,0,1)}(A)\right)$ & $\operatorname{dim}_{\mathbb{K}}\left(\operatorname{Der}_{(0,1,0)}(A)\right)$ \\
\hline$A s_{3}^{1}$ & 3 & 3 & 6 & 4 & 3 & 3 \\
\hline$A s_{3}^{2}$ & 3 & 3 & 6 & 4 & 3 & 3 \\
\hline$A s_{3}^{3}$ & 3 & 3 & 3 & 3 & 3 & 3 \\
\hline$A s_{3}^{4}$ & 5 & 3 & 3 & 3 & 6 & 3 \\
\hline$A s_{3}^{5}$ & 3 & 3 & 0 & 0 & 3 & 3 \\
\hline$A s_{3}^{6}$ & 3 & 5 & 3 & 3 & 3 & 6 \\
\hline$A s_{3}^{7}$ & 3 & 3 & 0 & 0 & 0 & 0 \\
\hline$A s_{3}^{8}$ & 5 & 2 & 0 & 0 & 3 & 0 \\
\hline$A s_{3}^{9}$ & 2 & 5 & 0 & 0 & 0 & 3 \\
\hline$A s_{3}^{10}$ & 3 & 3 & 0 & 0 & 0 & 0 \\
\hline$A s_{3}^{11}$ & 3 & 3 & 3 & 3 & 3 & 3 \\
\hline$A s_{3}^{12}$ & 3 & 3 & 0 & 0 & 0 & 0 \\
\hline$A s_{3}^{13}$ & 3 & 3 & 0 & 0 & 0 & 0 \\
\hline$A s_{3}^{14}$ & 3 & 3 & 0 & 0 & 0 & 0 \\
\hline$A s_{3}^{15}$ & 5 & 2 & 0 & 0 & 3 & 0 \\
\hline$A s_{3}^{16}$ & 2 & 5 & 0 & 0 & 0 & 3 \\
\hline$A s_{3}^{17}$ & 3 & 3 & 3 & 3 & 3 & 3 \\
\hline$A s_{3}^{18}$ & 9 & 9 & 9 & 9 & 9 & 9 \\
\hline
\end{tabular}

Table 3: Contraction invariants of three-dimensional associative algebras. 


\begin{tabular}{|c|c|c|c|c|c|c|}
\hline $\overrightarrow{d e g}$ & $A s_{3}^{1}$ & $A s_{3}^{2}$ & $A s_{3}^{3}$ & $A s_{3}^{4}$ & $A s_{3}^{5}$ & $A s_{3}^{6}$ \\
\hline$A s_{3}^{1}$ & - & $\operatorname{Com}(A)$ & $r_{3}(A)$ & $\operatorname{Aut}(A)$ & $Z(A)$ & $H^{1}(A, A)$ \\
\hline$A s_{3}^{2}$ & $\operatorname{Aut}(A)$ & - & $r_{3}(A)$ & $H^{2}(A, A)$ & $Z(A)$ & $H^{1}(A, A)$ \\
\hline$A s_{3}^{3}$ & $\sqrt{ }$ & $H^{2}(A, A)$ & - & $H^{2}(A, A)$ & $Z^{2}(A, A)$ & $\operatorname{Com}(A)$ \\
\hline$A s_{3}^{4}$ & $R(A)$ & $L(A)$ & $R(A)$ & - & $H^{2}(A, A)$ & $\operatorname{Der}_{(1,1,0)}(A)$ \\
\hline$A s_{3}^{5}$ & $O(A)$ & $O(A)$ & $\operatorname{Aut}(A)$ & $\operatorname{Aut}(A)$ & - & $\operatorname{Der}(A)$ \\
\hline$A s_{3}^{6}$ & $R(A)$ & $Z(A)$ & $R(A)$ & $\operatorname{Der}_{(1,0,1)}(A)$ & $\mathrm{d}\left(A^{2}\right)$ & - \\
\hline$A s_{3}^{7}$ & $\sqrt{ }$ & $Z(A)$ & $\sqrt{ }$ & $\sqrt{ }$ & $Z(A)$ & $\sqrt{ }$ \\
\hline$A s_{3}^{8}$ & $\operatorname{Der}_{(1,1,0)}(A)$ & $\operatorname{Der}_{(1,1,0)}(A)$ & $\operatorname{Aut}(A)$ & $\operatorname{Aut}(A)$ & $Z(A)$ & $\operatorname{Der}(A)$ \\
\hline$A s_{3}^{9}$ & $\operatorname{Der}_{(1,0,1)}(A)$ & $\operatorname{Der}_{(1,0,1)}(A)$ & $\operatorname{Aut}(A)$ & $\operatorname{Der}(A)$ & $H^{2}(A, A)$ & $\operatorname{Aut}(A)$ \\
\hline$A s_{3}^{10}$ & $H^{2}(A, A)$ & $\operatorname{Aut}(A)$ & $\operatorname{Aut}(A)$ & $\operatorname{Der}(A)$ & $\operatorname{Com}(A)$ & $\operatorname{Com}(A)$ \\
\hline$A s_{3}^{11}$ & $R(A)$ & $L(A)$ & $R(A)$ & $\operatorname{Com}(A)$ & $Z(A)$ & $\operatorname{Com}(A)$ \\
\hline$A s_{3}^{12}$ & $\sqrt{ }$ & $\operatorname{Com}(A)$ & $\sqrt{ }$ & $H^{1}(A, A)$ & $\operatorname{Com}(A)$ & $Z(A)$ \\
\hline$A s_{3}^{13}$ & $\sqrt{ }$ & $\operatorname{Com}(A)$ & $\sqrt{ }$ & $Z(A)$ & $\operatorname{Com}(A)$ & $\operatorname{Com}(A)$ \\
\hline$A s_{3}^{14}$ & $\sqrt{ }$ & $Z(A)$ & $\sqrt{ }$ & $\operatorname{Com}(A)$ & $Z(A)$ & $\operatorname{Com}(A)$ \\
\hline$A s_{3}^{15}$ & $\operatorname{Der}_{(1,1,0)}(A)$ & $\operatorname{Der}_{(1,1,0)}(A)$ & $\operatorname{Der}_{(1,1,0)}(A)$ & $\sqrt{ }$ & $Z(A)$ & $\operatorname{Der}_{(1,1,0)}(A)$ \\
\hline$A s_{3}^{16}$ & $\operatorname{Der}_{(1,0,1)}(A)$ & $\operatorname{Der}_{(1,0,1)}(A)$ & $\operatorname{Der}_{(1,0,1)}(A)$ & $\operatorname{Der}_{(1,0,1)}(A)$ & $Z(A)$ & $\sqrt{ }$ \\
\hline$A s_{3}^{17}$ & $\sqrt{ }$ & $\operatorname{Com}(A)$ & $\sqrt{ }$ & $H^{1}(A, A)$ & $H^{2}(A, A)$ & $H^{1}(A, A)$ \\
\hline$A s_{3}^{18}$ & $R(A)$ & $L(A)$ & $\operatorname{Aut}(A)$ & $\operatorname{Com}(A)$ & $S A(A)$ & $d\left(A^{2}\right)$ \\
\hline $\overrightarrow{d e g}$ & $A s_{3}^{7}$ & $A s_{3}^{8}$ & $A s_{3}^{9}$ & $A s_{3}^{10}$ & $A s_{3}^{11}$ & $A s_{3}^{12}$ \\
\hline$A s_{3}^{1}$ & $H^{2}(A, A)$ & $O(A)$ & $d^{2}(A)$ & $R(A)$ & $\operatorname{Der}(A)$ & $L(A)$ \\
\hline$A s_{3}^{2}$ & $R(A)$ & $L(A)$ & $H^{2}(A, A)$ & $\mathrm{d}^{2}(A)$ & $\operatorname{Aut}(A)$ & $R(A)$ \\
\hline$A s_{3}^{3}$ & $Z(A)$ & $\operatorname{Com}(A)$ & $R(A)$ & $L(A)$ & $O(A)$ & $S A(A)$ \\
\hline$A s_{3}^{4}$ & $\mathrm{~d}^{2}(A)$ & $Z(A)$ & $H^{2}(A, A)$ & $L(A)$ & $O(A)$ & $\operatorname{Aut}(A)$ \\
\hline$A s_{3}^{5}$ & $R(A)$ & $L(A)$ & $R(A)$ & $R(A)$ & $\operatorname{Aut}(A)$ & $S A(A)$ \\
\hline$A s_{3}^{6}$ & $H^{2}(A, A)$ & $L(A)$ & $R(A)$ & $L(A)$ & $O(A)$ & $S A(A)$ \\
\hline$A s_{3}^{7}$ & - & $Z(A)$ & $Z(A)$ & $\sqrt{ }$ & $\operatorname{Aut}(A)$ & $O(A)$ \\
\hline$A s_{3}^{8}$ & $S A(A)$ & - & $R(A)$ & $R(A)$ & $O(A)$ & $H^{2}(A, A)$ \\
\hline As & $L(A)$ & $L(A)$ & - & $L(A)$ & $\operatorname{Der}(A)$ & $\operatorname{Der}(A)$ \\
\hline$A s_{3}^{10}$ & $S A(A)$ & $H^{1}(A, A)$ & $O(A)$ & - & $H^{2}(A, A)$ & $\operatorname{Aut}(A)$ \\
\hline$A s_{3}^{11}$ & $H^{1}(A, A)$ & $H^{2}(A, A)$ & $H^{1}(A, A)$ & $\mathrm{d}^{2}(A)$ & - & $H^{2}(A, A)$ \\
\hline$A s_{3}^{12}$ & $H^{1}(A, A)$ & $H^{1}(A, A)$ & $\operatorname{Com}(A)$ & $\sqrt{ }$ & $\operatorname{Aut}(A)$ & - \\
\hline$A s_{3}^{13}$ & $\operatorname{Com}(A)$ & $Z(A)$ & $\operatorname{Der}_{(1,1,0)}(A)$ & $\sqrt{ }$ & $\sqrt{ }$ & $Z^{2}(A, A)$ \\
\hline$A s_{3}^{14}$ & $H(A, A)$ & $\operatorname{Com}(A)$ & $\operatorname{Com}(A)$ & $\sqrt{ }$ & $\sqrt{ }$ & $H^{2}(A, A)$ \\
\hline$A s_{3}^{15}$ & $R(A)$ & $\sqrt{ }$ & $\operatorname{Der}_{(1,1,0)}(A)$ & $R(A)$ & $\operatorname{Aut}(A)$ & $R(A)$ \\
\hline$A s_{3}^{16}$ & $L(A)$ & $L(A)$ & $\sqrt{ }$ & $L(A)$ & $\operatorname{Der}(A)$ & $L(A)$ \\
\hline$A s_{3}^{17}$ & $R(A)$ & $H^{2}(A, A)$ & $R(A)$ & $L(A)$ & $\operatorname{Aut}(A)$ & $Z^{2}(A, A)$ \\
\hline$A s_{3}^{18}$ & $O(A)$ & $Z(A)$ & $\operatorname{Der}(A)$ & $Z^{2}(A, A)$ & $H^{1}(A, A)$ & $H^{2}(A, A)$ \\
\hline $\overrightarrow{d e g}$ & $A s_{3}^{13}$ & $A s^{14}$ & $A s_{3}^{15}$ & $A s_{3}^{16}$ & $A s_{3}^{17}$ & $A s_{3}^{18}$ \\
\hline
\end{tabular}


Citation: Mohammed NF, Rakhimov IS, Said Husain SK (2017) On Contractions of Three-Dimensional Complex Associative Algebras. J Generalized Lie Theory Appl 11: 282. doi: 10.4172/1736-4337.1000282

Page 6 of 6

\begin{tabular}{|c|c|c|c|c|c|c|}
\hline$A s_{3}^{1}$ & $H^{1}(A, A)$ & $S A(A)$ & $H^{2}(A, A)$ & $O(A)$ & $S A(A)$ & $\sqrt{ }$ \\
\hline$A s_{3}^{2}$ & $L(A)$ & $\operatorname{Der}_{(1,0,0)}(A)$ & $\operatorname{Der}_{(0,1,1)}(A)$ & $S A(A)$ & $\mathrm{H}^{2}(A, A)$ & $\sqrt{ }$ \\
\hline As & $\operatorname{Aut}(A)$ & $\operatorname{Der}(A)$ & $\operatorname{Der}(A)$ & $\operatorname{Com}(A)$ & $H^{2}(A, A)$ & $\sqrt{ }$ \\
\hline$A s_{3}^{4}$ & $\operatorname{Der}^{2}(A)$ & $S A(A)$ & $Z(A)$ & $H^{1}(A, A)$ & $O(A)$ & $\sqrt{ }$ \\
\hline$A s_{3}^{5}$ & $H^{1}(A, A)$ & $S A(A)$ & $O(A)$ & $H^{1}(A, A)$ & $\operatorname{Der}(A)$ & $\sqrt{ }$ \\
\hline$A s_{3}^{6}$ & $\operatorname{Der}(A)$ & $\operatorname{Der}(A)$ & $H^{2}(A, A)$ & $Z(A)$ & $O(A)$ & $\sqrt{ }$ \\
\hline$A s_{3}^{7}$ & $S A(A)$ & $O(A)$ & $Z(A)$ & $Z(A)$ & $\operatorname{Aut}(A)$ & $\sqrt{ }$ \\
\hline$A s_{3}^{8}$ & $O(A)$ & $S A(A)$ & $H^{1}(A, A)$ & $H^{2}(A, A)$ & $\operatorname{Aut}(A)$ & $\sqrt{ }$ \\
\hline$A s_{3}^{9}$ & $\operatorname{Aut}(A)$ & $L(A)$ & $H^{1}(A, A)$ & $S A(A)$ & $O(A)$ & $\sqrt{ }$ \\
\hline$A s_{3}^{10}$ & $O(A)$ & $H^{2}(A, A)$ & $H^{2}(A, A)$ & $Z(A)$ & $O(A)$ & $\sqrt{ }$ \\
\hline$A s_{3}^{11}$ & $\operatorname{Der}(A)$ & $\operatorname{Aut}(A)$ & $Z(A)$ & $L(A)$ & $R(A)$ & $\sqrt{ }$ \\
\hline$A s_{3}^{12}$ & $S A(A)$ & $\operatorname{Aut}(A)$ & $Z(A)$ & $\operatorname{Com}(A)$ & $\operatorname{Aut}(A)$ & $\sqrt{ }$ \\
\hline$A s_{3}^{13}$ & - & $\sqrt{ }$ & $\operatorname{Der}_{(1,0,1)}(A)$ & $Z(A)$ & $\sqrt{ }$ & $\sqrt{ }$ \\
\hline$A s_{3}^{14}$ & $S A(A)$ & - & $H^{2}(A, A)$ & $H^{1}(A, A)$ & $\sqrt{ }$ & $\sqrt{ }$ \\
\hline$A s_{3}^{15}$ & $S A(A)$ & $O(A)$ & - & $R(A)$ & $\operatorname{Aut}(A)$ & $\sqrt{ }$ \\
\hline$A s_{3}^{16}$ & $S A(A)$ & $\operatorname{Aut}(A)$ & $L(A)$ & - & $\operatorname{Aut}(A)$ & $\sqrt{ }$ \\
\hline$A s_{3}^{17}$ & $\operatorname{Aut}(A)$ & $\operatorname{Aut}(A)$ & $Z(A)$ & $R(A)$ & & $\sqrt{ }$ \\
\hline$A s_{3}^{18}$ & $O(A)$ & $L(A)$ & $\operatorname{Der}(A)$ & $Z^{2}(A, A)$ & $H^{1}(A, A)$ & $R(A)$ \\
\hline
\end{tabular}

Table 4: All possibilities of degenerations of three-dimensional associative algebras.

\section{Theorem 4.2}

The rigid irreducible components of the variety $A_{3}(\mathbb{C})$ are generated by the algebras $A s_{3}^{2}, A s_{3}^{5}, A s_{3}^{7}, A s_{3}^{12}, A s_{3}^{13}, A s_{3}^{15}$ and $A s_{3}^{16}$ and with the dimensions:

$$
\begin{aligned}
& C_{1}=\overline{\operatorname{Orb}\left(A s_{3}^{2}\right),}, \quad \operatorname{dim}_{\mathbb{C}} C_{1}=5, \\
& C_{2}=\overline{\operatorname{Orb}\left(A s_{3}^{5}\right)}, \quad \operatorname{dim}_{\mathbb{C}} C_{1}=5, \\
& C_{3}=\overline{\operatorname{Orb}\left(A s_{3}^{7}\right)}, \quad \operatorname{dim}_{\mathbb{C}} C_{2}=7, \\
& C_{4}=\overline{\operatorname{Orb}\left(A s_{3}^{12}\right)}, \quad \operatorname{dim}_{\mathbb{C}} C_{3}=7, \\
& C_{5}=\overline{\operatorname{Orb}\left(A s_{3}^{13}\right)}, \quad \operatorname{dim}_{\mathbb{C}} C_{4}=9, \\
& C_{6}=\overline{\operatorname{Orb}\left(A s_{3}^{15}\right)}, \quad \operatorname{dim}_{\mathbb{C}} C_{5}=7, \\
& C_{7}=\overline{\operatorname{Orb}\left(A s_{3}^{16}\right)}, \quad \operatorname{dim}_{\mathbb{C}} C_{6}=7,
\end{aligned}
$$

As a consequence, $\operatorname{dim}_{\mathbb{C}} A_{3}(\mathbb{C})=9$.

\section{References}

1. Segal IE (1961) A class of operator algebras which are determined by groups. Duke Mathematical Journal 18: 221-265.

2. Inönu E, Eugene PW (1953) On the contraction of groups and their representations. Proceedings of the National Academy of Sciences 39: 510524.

3. Gabriel P (1975) Finite representation type is open. Representations of algebras, Springer, Berlin- Heidelberg, pp: 132-155.

4. Mazzola G (1979) The algebraic and geometric classification of associative algebras of five. Manuscripta Math 27: 81-101.

5. Makhlouf A, Goze M (1990) Classification of rigid associative algebras in lowdimensions. Preprint, ULP-IRMA, pp: 425-238.
6. Makhlouf A (1993) The irreducible components of the nilpotent associative algebras. Revista Matematica Complutense 6: 27-40.

7. Makhlouf A (2007) A comparison of deformations and geometric study of varieties of associative algebras. International Journal of Mathematics and Mathematical Sciences Article ID 18915, p: 24.

8. Rakhimov IS, Mohd Atan KA (2012) On contractions and invariants of Leibniz algebras. Bull Malays Math Sci Soc 35: 557-565.

9. Pierce RS (1982) Associative Algebras. Springer New York.

10. Grunewald F, O'Halloran J (1988) Varieties of nilpotent Lie algebras of dimension less than six. Journal of Algebra 112: 315-325.

11. Nesterenko M, Popovych R (2006) Contractions of low-dimensional Lie algebras. Journal of Mathematical Physics 47: 123515

12. Burde D (2005) Degenerations of $n$-dimensional nilpotent Lie algebras Communications in Algebra 33: 1259-1277.

13. Rakhimov IS, Rikhsiboev RM, Basri W (2010) Classification of 3-dimensional complex diassociative algebras. Malaysian Journal of Mathematical Sciences 4: $241-254$

14. Rakhimov IS, Riskhboev IM, Basri W - Complete list of low-dimensional complex associative algebras, arXiv:0910.0932v2, math.RA.

15. Fiidow MA, Rakhimov IS, Said Hussain SK (2016) Derivations and Centroids of Associative algebras. IEEE Conference Publications, pp: 227-232.

16. Rakhimov IS, Said Husain ShK, Abdulkadir A (2016) On Generalized Derivations of finite dimensional Associative algebras, FEIIC International Journal of Engineering and Technology 13: 121-126. 